
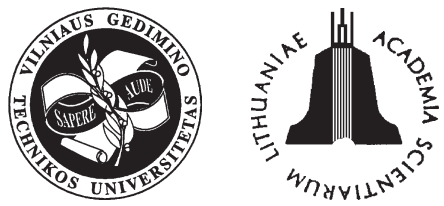

ISSN 1648-4142 TRANSPORT

http:/www.vtu.lt/english/editions

TRANSPORT-2005, Vol XX, No 1, 51-54

\title{
HAULAGE TRUCKS MODEL WITH FOUR ELECTRIC SEPARATE WHEEL DRIVES
}

\author{
Rafal Setlak \\ Dept of Electrical Engineering in Transport, Silesian University of Technology, \\ Akademicka 10, 44-100 Gliwice, Poland, tel/fax: +48 322371258
}

Received 2004-11-01; accepted 2004-12-03

\begin{abstract}
This article contains a course of work of the construction of a vehicle model that has four electrical motors built into each wheel. During the project two models of a vehicle were constructed (Figs 3,5). A microprocessor based control system has also been designed and built. A vehicle is controlled by a steering unit (Fig 8 ) which contains a steering wheel with a force feedback system, push buttons, an accelerator and a brake. The connection between a steering unit and a model is realized by interface RS-485. The driving motors are dc motors with permanent magnets. Power supply consists of an acid battery located in the vehicle. The vehicle control system is divided into two parts. The first part is built into the vehicle (Fig 7) and operates as a vehicle main control system and the second is built into a steering unit and operates as the main control steering system.
\end{abstract}

Keywords: automotive electronic systems, electric vehicle, in-wheel motor, haulage truck, four-wheel drive, traction motor drives, mining haul trucks.

\section{Introduction}

In Polish mines over 1000 special trucks with a combustion engine only operate at present. The total installed power of all trucks is equal to $150 \mathrm{MW}$.

A traditional haulage truck (Fig 1) consists of two movable parts joined to each other. These parts are:

- driving module (which contains a combustion engine),

- dump trailer.

A haulage truck can turn only in the hydraulic joint between a driving module and its trailer and all the wheels cannot be turned horizontally in relation to the vehicle body. Both parts can revolve mutually to ensure a better contact of wheels with the bed rock during driving over obstacles or slopes.

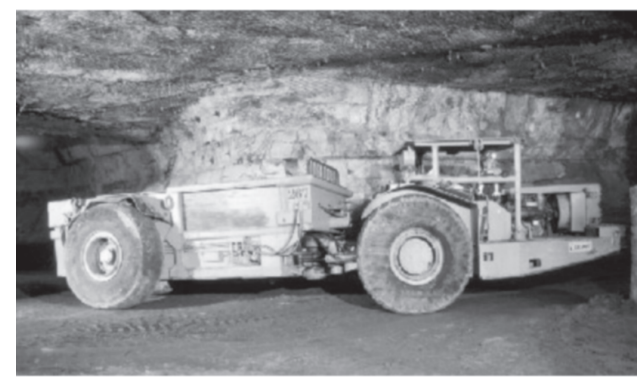

Fig 1. Traditional haulage truck with a combustion engine during work
In recent years there were some attempts to build electrical drives into the haulage trucks. At first electric motors were supplied through unrolled wires or contact lines in a mine (Fig 2).

Replacing a combustion engine with an electrical motor fed by batteries was also tested and the results were promising. The best solution, in the author's opinion, is to substitute a traditional drive system with a system consisting of electrical motors built into all four wheels of the vehicle.

This solution calls for new design philosophy of the drive system. All wheels must be controlled. The task of new controls should take into consideration the following problems: synchronization, protection against wrong operation and driving. Controls also should perform a driving program under different

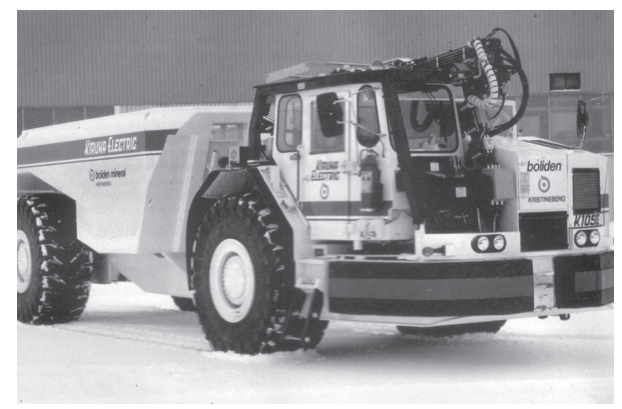

Fig 2. Haulage truck supplied by contact lines in a mine 
conditions which could occur at the working site (narrow footwall drift, different road profiles, sharp turns, steep slopes, changeable hardness and dampness of the drift) [1-6].

\section{Design brief}

Design requirements are: to achieve flexible and module design which makes the modification, repair or replacements of drive elements as quick as possible. Also the development of new design with additional elements should be possible. This solution enables utilization control system which could be used in any model of a vehicle. There is obviously possibility to add modules which could be used in the future.

\section{The two generations of haulage truck models}

The first-generation model has been built to scale 1:23. It is composed of four step motors SH 200 and controls supported by four microcontrollers AT89C2051 for each wheel (Fig 3). The real haulage truck size is shown in Fig 4 and the paper's author is shown here for comparison purposes.

The program is written in the Visual Basic. Haulage truck model contains autonomic battery $(12 \mathrm{~V}$, $1,2 \mathrm{Ah}$ ). The communication between a model and PC computer is accomplished by using RS232 standard. This model was useful for the observation of operation of a control system and driving modules.

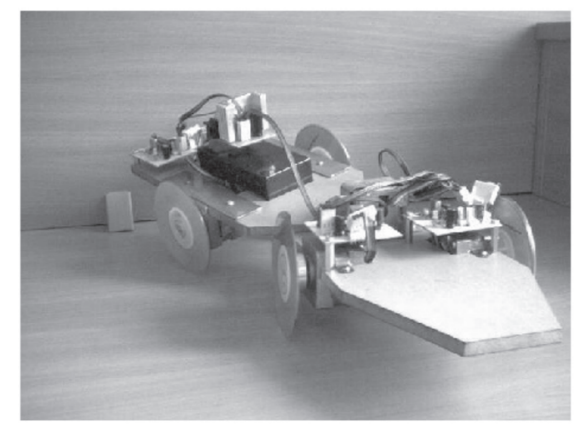

Fig 3. Electric vehicle model the first generation with four motors built into wheels

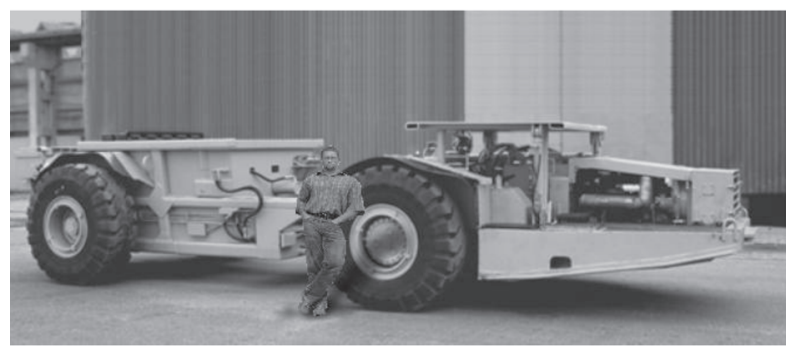

Fig 4. Real haulage truck, the author is shown in order to assess the truck size
The control algorithm has also been used in the second generation of a model (Fig 5) which has been created to scale 1:18. The second model is composed of four dc motors with permanent magnets $\left(\mathrm{U}_{\mathrm{N}}=3,6 \mathrm{~V}, \mathrm{~T}_{\mathrm{MAX}}=2 \mathrm{Nm}, \mathrm{n}_{\mathrm{N}}=230 \mathrm{rpm}\right)$. The double planetary gear provides high driving torque and low rotational speed. Each of the used driving motors has its own control module composed of microcontrollers Atmel AT90S2313 which belong to AVR processors family.

Series transmission system UART is indispensable to receive instructions which are sent by Vehicle Main Control System (VMCS) installed in a vehicle. The communication between control systems in a model is accomplished by using RS232. It creates Internal Control Area Network (I-CAN) of the vehicle which allows transmitting signals to driving motors and data from position measuring systems.

For proper and reliable operation the WATCHDOG unit is used. In case when VMCS does not receive data, microcontroller RESET occurs and software restarts again and turns off the drive motor. Protection is used in the case of losing connection between VMCS and Main Controller of Steering System (MCSS) or motor failure.

\section{Power supply and control system}

Power supply consists of an acid-type battery (Fig 6) located in a vehicle (with rated voltage $6 \mathrm{~V}$, capacity $12 \mathrm{Ah}$ ) and voltage regulator LM2940CT-5. This voltage regulator has temperature and overcurrent protection.

The Vehicle Control System becomes divided into two parts. The first part is built in a vehicle (Fig 7) and operates as VMCS and the second is built in Steering Unit and operates as MCSS. VMCS is responsible for the communication and measuring the analog data from input devices controlling UART.

MCSS is the processor ATmega128, identical as

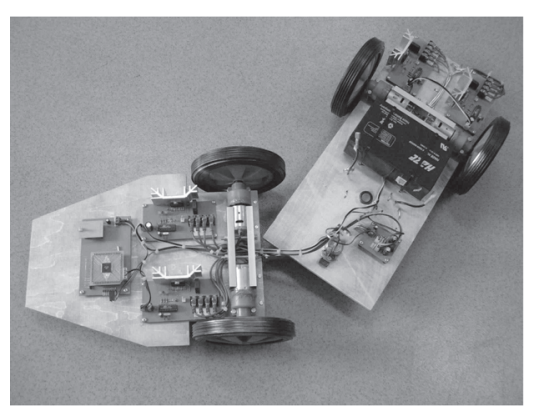

Fig 5. Electric vehicle models the second generation with four DC motors with double planetary gears built into wheels 


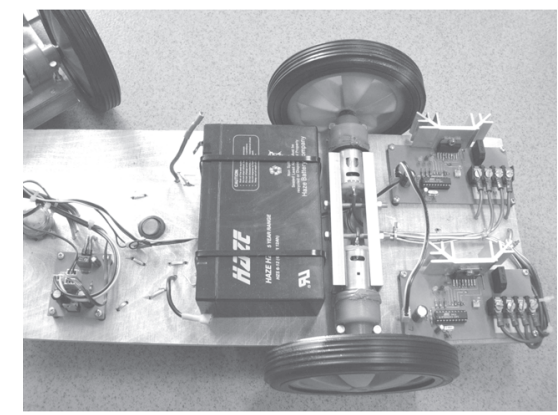

Fig 6. Power supply in an electric vehicle of the second generation model

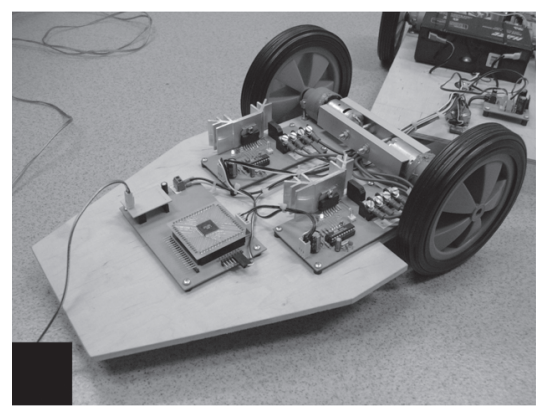

Fig 7. Control system VMCS in an electric vehicle of the second generation model

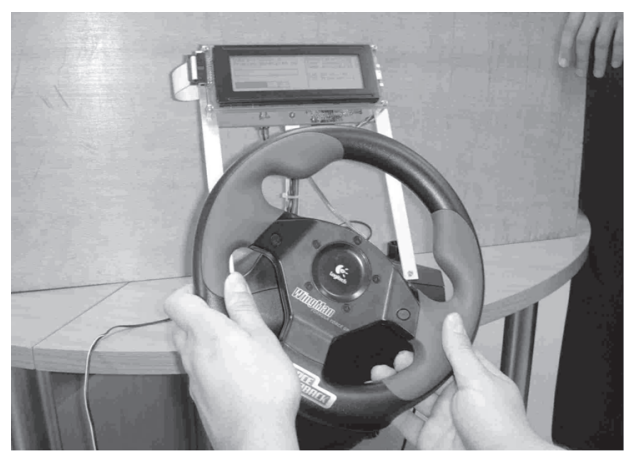

Fig 8. Wheel with ForceFeedback system with digital display

one built in a vehicle. Its main task is to communicate with a vehicle, data transmission and operation as per steering algorithm. The reason for locating of the work algorithms in Steering Unit comes from previous research, conducted with the first generation model of a vehicle. If algorithms were put in the vehicle, processor communication between Steering Unit and MCSV would slow down.

Steering Unit (Fig 8) is the input device of a vehicle. It contains a steering wheel with push buttons (forward drive, reverse, stop), an accelerator and brake.

Actual data like battery voltage, turning angle, speed, forward drive, reverse drive, temperature are

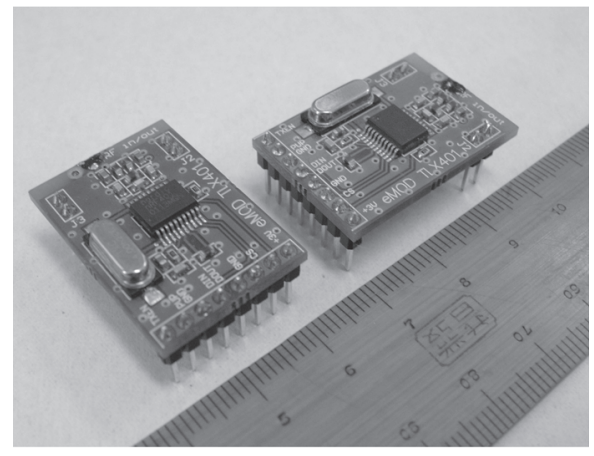

Fig 9. Radio frequency communicator modules TLX401

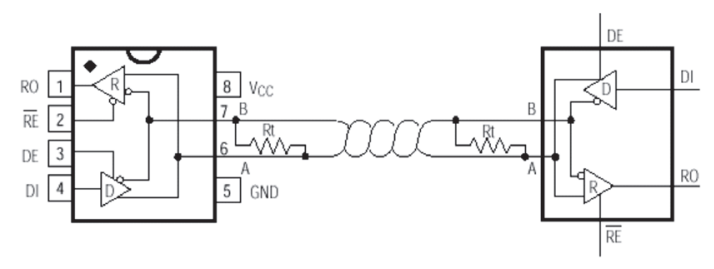

Fig 10. Wire transmission system in RS-485 standard

shown on the display panel of $240 \times 64$ points resolution, it is located above the wheel. Analog data are represented as analog rulers. This method is more driver-friendly.

A wheel has a "ForceFeedback" system with a direct current motor of $24 \mathrm{~V}$. Feedback controls the turning angle and tries to set up a steering wheel in accordance with the angle of articulated joint. When power supply and Steering Unit are on, the steering wheel is put at the same angle as the vehicle model. This makes it impossible to straighten the vehicle after power supply is switched on. The main motor of "ForceFeedback" system is controlled by MCSS. Microcontroller AT2313 PWM unit also controls rotational speed of driving motors in each wheel. The PWM unit is configured with 10-bit resolution (steering voltage may assume 1024 different values). PWM outputs control the bridge circuit L298. In this topology there are two transistor bridges and logic control unit.

Modules which might later be added to MCSV design are laser position measuring systems and radio frequency communicator TLX401 (Fig 9). This would replace a wire transmission system in RS-485 standard.

RS-485 interface (Fig 10) which has been set up is characterized by simple structure, dependability and high speed data transmission (up to $4 \mathrm{Mbit} / \mathrm{s}$ ) and spiral structure with length up to $1200 \mathrm{~m}$. This standard is similar to CAN-bus which is at present widely used in car communication systems. 


\section{Software}

We used BASCOM-AVR software for programming a vehicle model for microcontrollers AVR and BASCOM-8051 for AT89C2051. BASIC language has been chosen for several important reasons. The simplicity and characteristic features of AVR processors make possible fast modernization of steering software. Debugging of steering programs is easy because of built-in simulation procedures in BASCOM compilers. The data transmission protocol between MCSV and MCSS is supported by protocol S.N.A.P., this simplifies the communication between all parts of a vehicle system. Free software, source codes and tools facilitate PC programming.

\section{Control algorithm}

The following parameters are taken into consideration by the control algorithm: axle base, wheel track, turning angle and vehicle speed. On the basis of this data adequate rotational speeds for the particular wheels are calculated. Turning force in articulated joint during drive requires rotational speeds correction separate for each driving motor. When the model drives forward there are corrections of all wheels. Forward driving algorithm depending on the change of a turning angle controls the speeds of vehicle wheels. A wheel which should go by a shorter way reduces rotational speed and the second wheel on the same axle should accelerate speed. The differences between speeds are proportional to the value of the difference of demanded turning angle and measured real vehicle angle. While driving backwards the algorithm is similar with the difference that a rear axle and the front wheels are moving straight. This kind of motion continues till the turning angle is achieved. Another algorithm controls the turning of a non-moving vehicle. In this case MCSS reverses rotational speeds of wheels in each axle dependent on drive direction If a forward direction is chosen, it will be front axle, with a backward direction it will be rear axle. If the turning of the vehicle is performed in a neutral gear both axles react at the same time.

\section{Conclusion}

The conducted research of the first generation model has shown that the best control is achieved by putting a microcontroller with a steering algorithm into a vehicle and equipping driving motors with their own microprocessor control systems. With continued research (second-generation model) we changed our opinion on the design. In the new design one microprocessor is responsible for measuring analog data and controlling data transmission, whereas the second one built in MCSS takes care of a steering algorithm and supervision system.

In order to maintain correct operation the system has to use fast bi-directional data transmission between its parts. In the beginning we thought about communication by using radio frequency $433 \mathrm{MHz}$. For that purpose we have TLX401 communicators which operate at $433,93 \mathrm{MHz}$ or $434,33 \mathrm{MHz}$ and has data range up to $800 \mathrm{~m}$ with $19,2 \mathrm{kbit} / \mathrm{s}$ transmission speed. Testing the present design we have found that there is no correct transmission between MCSV and MCSS. The reason of this is known. It is uncorrected synchronisation of transmission data start of microprocessors. During the next stages of the vehicle development we want to work on this problem and use special decoding and filtering algorithms.

The preliminary design of a vehicle model makes an assumption about using optical measuring systems to describe the position of vehicle parts. Our experiments showed that these systems are very sensitive during assembly. At present we do not use these sensors, but we control system with processors of ' 51 family (which can be adapted to optical measuring systems). In the next stages of the development we will use these optical measuring units.

\section{References}

1. Doliński, J. Microcontrollers AVR in practice (Mikrokontrolery AVR w praktyce), Warszawa, 2003.

2. Starecki, T. Microcontrollers 8051 in practice (Mikrokontrolery $8051 \mathrm{w}$ praktyce), Warszawa, 2002.

3. Hadam, P. Designing of microprocesser systems (Projektowanie systemów mikroprocesorowych), Warszawa, 2004.

4. ATMEL.: 8-bit Microcontroller with 2K Bytes of InSystem Programmable Flash. AT90S2313.

5. ATMEL.: 8-bit Microcontroller with 128K Bytes of InSystem Programmable Flash. AT9mega128.

6. ATMEL.: 8-bit Microcontroller with 2K Bytes Flash. AT89C2051. 